\title{
Synthesis of Substituted 3,4-Dialkyl-2,6-Diaryl- Piperidin-4-ol Derivatives and Their Conformational Study
}

\author{
MARUTHAVANAN T and VENKATESAN P* \\ Department of Chemistry, Sona College of Technology, \\ Salem - 636 005, India \\ *Department of Chemistry, Mahendra Institute of Technology, \\ Namakkal - 637 503, India \\ venkatesanps@yahoo.co.in
}

Received 23-Nov-2011; Accepted 15-Jan-2012

\begin{abstract}
The synthesis of series of 3,4-dialkyl-2,6-diaryl-piperidin-4-ol derivatives (7-12) by reduction of cis-3-alkyl-2,6-diarylpiperidin-4-one (1-6) using Grignard reagent has been reported. The synthesized heterocycles have been characterized on basis of spectroscopic data and their conformation has also been discussed.
\end{abstract}

Keywords: Piperidine, Grignard Reagent, Conformation, Spectra.

\section{Introduction}

2,6-disubstituted-piperidine derivatives are regarded as an important building block of many alkaloid natural products, therapeutic drugs and they serve a role as key intermediates for synthesis of various organic compounds including thiazolidones, oxirane, thiazine, etc [1-4]. The substituted piperidine derivatives are found to possess of various antimicrobial activities including anti-inflammatory, anti-tuberculosis, antipyretic, antibacterial, antifungal, etc [5, 6]. The antimicrobial activities are improved or declined when the conformation of piperidine is swapped due to substitution on different position. Hence, analyzing the conformation of compound has become important in recent years. Accordingly, Our recent investigation has demonstrated that reduction of keto functional group at $\mathrm{C}(4)$ of piperidine moiety incorporated with phenyl, $p$-chlorophenyl and $p$-methoxyphenyl substituents and they exerts noteworthy conformational changes. 


\section{Experimental}

\section{General details}

Melting point of synthesised compounds was determined in open glass capillaries and was uncorrected. IR spectra were recorded on Perkin-Elmer 577 IR spectrophotometer using KBr pellets. ${ }^{1} \mathrm{H}$ spectra $(500 \mathrm{MHz})$ and ${ }^{13} \mathrm{C}$ spectra $(125 \mathrm{MHz})$ were recorded on Bruker AVANCE III $500 \mathrm{MHz}$ NMR Spectrometer in $\mathrm{CDCl}_{3}$ with tetramethyl silane as the internal standard and the chemical shifts were reported in ppm scale. Mass spectra were studied using JEOL GCMATE II mass spectrometer. The purity of the compounds was checked by thin layer chromatography on silica gel $60 \mathrm{~F}_{254}$ (Merck) and spots were developed using iodine vapour or ultraviolet light.

\section{General synthesis of 4-hydroxy-piperidine derivative (7-12)}

Grignard reagent $(0.01 \mathrm{~mol})$ was added slowly to a solution of appropriate piperidin-4-one $(0.005 \mathrm{~mol})$ in sodium dried ether and the mixture was refluxed until the completion of reaction. The reaction mixture was allowed to cool and poured into the mixture of crushed ice $(125 \mathrm{~g}), 100 \mathrm{ml}$ of water and 10-20 $\mathrm{ml}$ of Conc. $\mathrm{HCl}$ contained in a $500 \mathrm{ml}$ beaker. The hydrochloride thus obtained was filtered and dried at the pump. The dried hydrochloride was pasted with 2 or 3 drops of acetone and neutralized with aqueous ammonia followed by the addition of water. The tertiary alcohols obtained were chromatographed from benzenechloroform (3:1) mixture.

\section{Synthesis of (3R,4R)-3-ethyl-4-(propan-2-yl)-2,6-bisphenylpiperidin-4-ol (7)}

Reaction Time: 18 Hours; Yield: $74 \%$; $R$ value $=0.34\left(\mathrm{CHCl}_{3}\right.$-Benzene $\left.=9: 1\right)$; Pale yellow viscous oil; IR $\left(\mathrm{KBr}, v_{\max }, \mathrm{cm}^{-1}\right): 3404.13$ and $3309.62(\mathrm{NH}$ and $\mathrm{OH}), 3061(\mathrm{ArCH}), 1279$ and 1026 (C-O str); ${ }^{1} \mathrm{H}$ NMR $\left(500 \mathrm{MHz}, \mathrm{CDCl}_{3}\right): \delta$ 7.18-7.48 (m, $\left.10 \mathrm{H}, \mathrm{ArH}\right), 4.05-4.08(\mathrm{dd}$, $\left.1 \mathrm{H}, 6-\mathrm{H}_{\mathrm{a}}\right), 3.93-3.97\left(\mathrm{~d}, 1 \mathrm{H}, 2-\mathrm{H}_{\mathrm{a}}\right), 2.66-2.73\left(\mathrm{~m}, 1 \mathrm{H}, 5-\mathrm{H}_{\mathrm{a}}\right), 2.62-2.65\left(\mathrm{dd}, 1 \mathrm{H}, 5-\mathrm{H}_{\mathrm{e}}\right), 2.58-$ $2.60\left(\mathrm{~m}, 1 \mathrm{H}, 3-\mathrm{H}_{\mathrm{a}}\right), 1.69(\mathrm{br}, 2 \mathrm{H}, \mathrm{NH}$ and $\mathrm{OH}), 1.05-1.09(\mathrm{~m}, 1 \mathrm{H}, 4-\mathrm{CH}), 0.94(\mathrm{t}, 3 \mathrm{H}, 4-$ $\left.\mathrm{CH}_{3}\right), 0.75\left(\mathrm{~m}, 2 \mathrm{H}, 3-\mathrm{CH}_{2}\right), 0.44\left(\mathrm{t}, 3 \mathrm{H}, 3-\mathrm{CH}_{3}\right) ;{ }^{13} \mathrm{C} \mathrm{NMR}\left(125 \mathrm{MHz}, \mathrm{CDCl}_{3}\right): \delta 126.05-$ 132.44, 68.15, 64.06, 62.01, 56.14, 47.99, 38.68, 18.05, 16.13, 10.99; HRMS: $323.46\left(\mathrm{M}^{+}\right)$ (Calcd. for $\mathrm{C}_{22} \mathrm{H}_{29} \mathrm{NO}$ : 323.47).

\section{Synthesis of (3R,4R)-3-ethyl-4-(propan-2-yl)-2,6-bis(4-chloro)phenylpiperidin-4-ol} (8)

Reaction Time: 21 Hours; Yield: $74.9 \%$; $R f$ Value $0.31\left(\mathrm{CHCl}_{3}\right.$-Benzene $\left.=9: 1\right)$; Pale yellow viscous oil; IR (KBr, $\left.v_{\max }, \mathrm{cm}^{-1}\right): 3350(\mathrm{NH}$ and $\mathrm{OH}), 2960(\mathrm{ArCH}), 1292$ and 1015 (C-O str), $765(\mathrm{ArCl}) ;{ }^{1} \mathrm{H}$ NMR $\left(500 \mathrm{MHz}, \mathrm{CDCl}_{3}\right)$ : $\delta$ 7.26-7.44 (m, 8H, ArH), 4.03-4.06 $\left(\mathrm{dd}, 1 \mathrm{H}, 6-\mathrm{H}_{\mathrm{a}}\right), 3.69-3.72\left(\mathrm{~d}, 1 \mathrm{H}, 2-\mathrm{H}_{\mathrm{a}}\right), 2.62-2.69\left(\mathrm{~m}, 5-\mathrm{H}_{\mathrm{a}}\right), 2.55-2.59\left(\mathrm{dd}, 1 \mathrm{H}, 5-\mathrm{H}_{\mathrm{e}}\right), 2.49-$ $2.54\left(\mathrm{~m}, 1 \mathrm{H}, 3-\mathrm{H}_{\mathrm{a}}\right), 1.65$ (br, 2H, NH and $\left.\mathrm{OH}\right), 0.78-0.95$ (m, 7H, 4-alkyl), 0.75 (m, 2H, 3$\left.\mathrm{CH}_{2}\right), 0.45\left(\mathrm{t}, 3 \mathrm{H}, 3-\mathrm{CH}_{3}\right) ;{ }^{13} \mathrm{C} \mathrm{NMR}\left(125 \mathrm{MHz}, \mathrm{CDCl}_{3}\right): \delta$ 127.84-130.86, 68.16, 66.00, 63.07, 58.44, 51.45, 38.91, 18.05, 16.08, 14.05, 10.95; HRMS: $392.54\left(\mathrm{M}^{+}\right)(\mathrm{Calcd}$. for $\mathrm{C}_{22} \mathrm{H}_{27} \mathrm{Cl}_{2} \mathrm{NO}$ : 392.36).

Synthesis of (3R,4R)-3-ethyl-4-(propan-2-yl)-2,6-bis(4-methoxy)phenyl-piperidin-4ol (9) 
Reaction Time: 20 Hours; Yield: $79.2 \%$; $R f$ Value $0.58\left(\mathrm{CHCl}_{3}-\mathrm{Benzene}=9: 1\right)$; Pale yellow viscous oil; IR ( $\left.\mathrm{KBr}, v_{\max }, \mathrm{cm}^{-1}\right): 3345(\mathrm{NH}$ and $\mathrm{OH}), 3059(\mathrm{ArCH}), 1300$ and 1034 (C-O str); ${ }^{1} \mathrm{H}$ NMR (500 MHz, $\mathrm{CDCl}_{3}$ ): $\delta$ 7.39-7.43 and 6.90-6.95 (m, 8H, ArH), 4.06-4.09 $\left(\mathrm{dd}, 1 \mathrm{H}, 6-\mathrm{H}_{\mathrm{a}}\right), 3.86,3.83\left(\mathrm{~s}, 6 \mathrm{H}, \mathrm{Ar}-\mathrm{OCH}_{3}\right), 3.71\left(\mathrm{~d}, 1 \mathrm{H}, 2-\mathrm{H}_{\mathrm{a}}\right), 2.58-2.78\left(\mathrm{~m}, 3 \mathrm{H}, 5-\mathrm{H}_{\mathrm{a}}, 5 \mathrm{H}_{\mathrm{e}}\right.$ and $\left.3-\mathrm{H}_{\mathrm{a}}\right), 1.59(\mathrm{br}, 2 \mathrm{H}, \mathrm{NH}$ and $\mathrm{OH}), 0.94-1.10\left(\mathrm{~m}, 7 \mathrm{H}, 4\right.$-alkyl), $0.79\left(\mathrm{t}, 1 \mathrm{H}, 3-\mathrm{CH}_{3}\right) ;{ }^{13} \mathrm{C}$ NMR (125 MHz, $\mathrm{CDCl}_{3}$ ): $\delta$ 114.52-123.42, 73.82, 64.27, 55.42, 51.35, 42.42, 31.87, 18.94, 10.66; HRMS: $383.25\left(\mathrm{M}^{+}\right)$(Calcd. for $\mathrm{C}_{24} \mathrm{H}_{33} \mathrm{NO}_{3}$ : 383.52).

\section{Synthesis of (3R,4R)-3,4-diethyl-2,6-bisphenylpiperidin-4-ol (10)}

Reaction Time: 20 Hours; Yield: $73.4 \%$; $f$ value $=0.58\left(\mathrm{CHCl}_{3}-\mathrm{Benzene}=9: 1\right)$; Pale yellow viscous oil; IR $\left(\mathrm{KBr}, v_{\max }, \mathrm{cm}^{-1}\right): 3352(\mathrm{NH}$ and $\mathrm{OH}), 3058(\mathrm{ArCH}), 1284$ and 1029 (C-O str); ${ }^{1} \mathrm{H}$ NMR (500 MHz, $\mathrm{CDCl}_{3}$ ): $\delta$ 7.47-7.53 and 7.24-7.35 (m, 10H, ArH), 4.25-4.28 (dd, 1H, 6- $\mathrm{H}_{\mathrm{a}}$ ), 3.95-3.97 (d, 1H, 2- $\left.\mathrm{H}_{\mathrm{a}}\right), 2.19(\mathrm{br}, 2 \mathrm{H}, \mathrm{NH}$ and $\mathrm{OH}), 1.75-1.78\left(\mathrm{dd}, 1 \mathrm{H}, 5-\mathrm{H}_{\mathrm{e}}\right.$ ), 1.81-1.82 (m, $\left.1 \mathrm{H}, 3-\mathrm{H}_{\mathrm{a}}\right), 1.64-1.68\left(\mathrm{~m}, 1 \mathrm{H}, 5-\mathrm{H}_{\mathrm{a}}\right), 1.05-1.13\left(\mathrm{~m}, 4 \mathrm{H}, 3\right.$ and $\left.4-\mathrm{CH}_{2}\right), 0.96(\mathrm{t}$, $\left.3 \mathrm{H}, 4-\mathrm{CH}_{3}\right), 0.49\left(\mathrm{t}, 3 \mathrm{H}, 3-\mathrm{CH}_{3}\right) ;{ }^{13} \mathrm{C} \mathrm{NMR}\left(125 \mathrm{MHz}, \mathrm{CDCl}_{3}\right): \delta 126.84-128.65,74.60$, 63.82, 56.87, 50.13, 44.72, 33.63, 19.00, 14.26, 7.94; HRMS: $309.24\left(\mathrm{M}^{+}\right)($Calcd. for $\left.\mathrm{C}_{21} \mathrm{H}_{27} \mathrm{NO}: 309.44\right)$.

\section{Synthesis of (3R,4R)-3,4-diethyl-2,6-bis(4-chloro)phenylpiperidin-4-ol (11)}

Reaction Time: 20 Hours; Yield: $82.1 \%$; $R f$ Value $0.4\left(\mathrm{CHCl}_{3}\right.$-Benzene $\left.=9: 1\right)$; Pale yellow viscous oil; IR (KBr, $\left.v_{\max }, \mathrm{cm}^{-1}\right): 3394(\mathrm{NH}$ and $\mathrm{OH}), 3031(\mathrm{ArCH}), 1288$ and $1010(\mathrm{C}-\mathrm{O}$ str), $723(\mathrm{ArCl}) ;{ }^{1} \mathrm{H}$ NMR $\left(500 \mathrm{MHz}, \mathrm{CDCl}_{3}\right): \delta$ 7.38-7.47 and 7.27-7.31 (m, 8H, ArH), 4.21-4.24 (dd, $\left.1 \mathrm{H}, 6-\mathrm{H}_{\mathrm{a}}\right), 3.92-3.94\left(\mathrm{~d}, 1 \mathrm{H}, 2-\mathrm{H}_{\mathrm{a}}\right), 1.75-1.78\left(\mathrm{dd}, 1 \mathrm{H}, 5-\mathrm{H}_{\mathrm{e}}\right), 1.70-1.73(\mathrm{~m}$, $\left.1 \mathrm{H}, 5-\mathrm{H}_{\mathrm{a}}\right), 1.62-1.67\left(\mathrm{~m}, 1 \mathrm{H}, 3-\mathrm{H}_{\mathrm{a}}\right), 1.47-1.50(\mathrm{br}, 2 \mathrm{H}, \mathrm{NH}$ and $\mathrm{OH}), 1.05-1.07(\mathrm{~m}, 2 \mathrm{H}, 4-$ $\left.\mathrm{CH}_{2}\right), 0.94\left(\mathrm{t}, 3 \mathrm{H}, 4-\mathrm{CH}_{3}\right), 0.80\left(\mathrm{~m}, 2 \mathrm{H}, 4-\mathrm{CH}_{2}\right), 0.48\left(\mathrm{t}, 3 \mathrm{H}, 3-\mathrm{CH}_{3}\right) ;{ }^{13} \mathrm{C} \mathrm{NMR}(125 \mathrm{MHz}$, $\left.\mathrm{CDCl}_{3}\right): \delta 128.06-130.48,74.40,62.93,56.14,50.20,44.72,33.58,18.90,14.61,7.92$; HRMS: $378.24\left(\mathrm{M}^{+}\right)$(Calcd. for $\mathrm{C}_{21} \mathrm{H}_{25} \mathrm{Cl}_{2} \mathrm{NO}$ : 378.34$)$.

\section{Synthesis of (3R,4R)-3,4-diethyl-2,6-bis(4-methoxy)phenylpiperidin-4-ol (12)}

Reaction Time: 22 Hours; Yield: $76.3 \%$; $R f$ Value $0.32\left(\mathrm{CHCl}_{3}-\mathrm{Benzene}=9: 1\right)$; Pale yellow viscous oil; IR $\left(\mathrm{KBr}, v_{\max }, \mathrm{cm}^{-1}\right): 3529(\mathrm{NH}$ and $\mathrm{OH}), 2962(\mathrm{ArCH}), 1299$ and 1033 (C-O str); ${ }^{1} \mathrm{H}$ NMR (500 MHz, $\left.\mathrm{CDCl}_{3}\right): \delta$ 7.36-7.60 and 6.85-7.01 (m, 8H, ArH), 4.16-4.19 $\left(\mathrm{dd}, 1 \mathrm{H}, 6-\mathrm{H}_{\mathrm{a}}\right), 3.865,3.833\left(\mathrm{~s}, 6 \mathrm{H}, \mathrm{Ar}-\mathrm{OCH}_{3}\right), 3.67-3.69\left(\mathrm{~d}, 1 \mathrm{H}, 2-\mathrm{H}_{\mathrm{a}}\right), 1.77-1.79(\mathrm{dd}, 1 \mathrm{H}$, 5- $\left.\mathrm{H}_{\mathrm{e}}\right), 1.68-1.76\left(\mathrm{~m}, 1 \mathrm{H}, 5-\mathrm{H}_{\mathrm{a}}\right), 1.62-1.67\left(\mathrm{~m}, 1 \mathrm{H}, 3-\mathrm{H}_{\mathrm{a}}\right), 1.472-1.51(\mathrm{br}, 2 \mathrm{H}, \mathrm{NH}$ and $\mathrm{OH})$, 0.934-1.10 (m, 4H, 3 and 4- $\left.\mathrm{CH}_{2}\right), 0.949\left(\mathrm{t}, 3 \mathrm{H}, 4-\mathrm{CH}_{3}\right), 0.508\left(\mathrm{t}, 1 \mathrm{H}, 3-\mathrm{CH}_{3}\right) ;{ }^{13} \mathrm{C} \mathrm{NMR}(125$ $\left.\mathrm{MHz}, \mathrm{CDCl}_{3}\right): \delta 113.40-114.36,127.63-131.08,74.66,63.16,56.20,50.35,44.84,33.67$, 19.04, 14.67, 7.96; HRMS: $369.29\left(\mathrm{M}^{+}\right)\left(\right.$Calcd. for $\left.\mathrm{C}_{23} \mathrm{H}_{31} \mathrm{NO}_{3}: 369.49\right)$.

\section{Results and Discussion}

The synthesis of target 3,4-dialkyl-2,6-diaryl-piperidin-4-ol derivatives (7-12) was accomplished by a Mannich reaction followed by reduction using Grignard reagent as shown in scheme 1. The parent compound, cis-2,6-diarylpiperidin-4-one derivatives (1-6) were synthesized by double mannich reaction using ketone, arylaldehyde and ammonium acetate in a stoichiometry of 1:2:1 ratio $[7,8]$. The product obtained was subjected to column chromatography using benzene-chloroform (3:1) eluent. 
<smiles>[R]c1ccc(C=O)cc1</smiles>

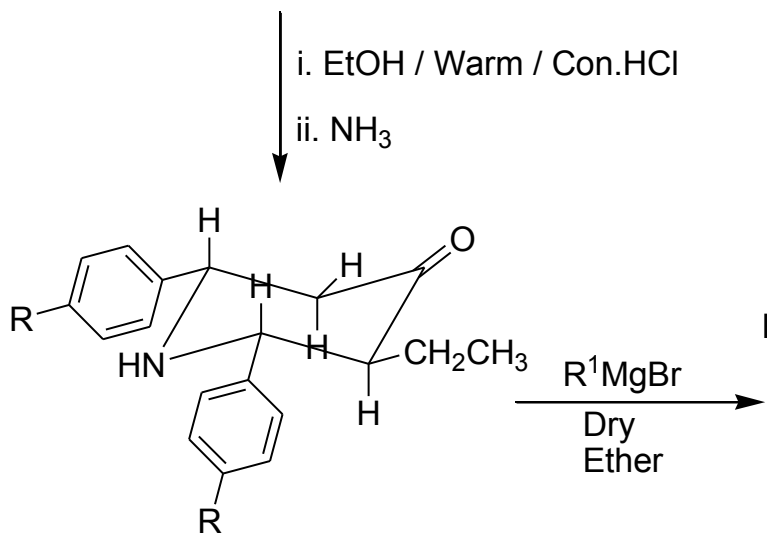

1 - 6
1, 7: $\mathrm{R}=\mathrm{H} ; \mathrm{R}^{1}=\mathrm{CH}\left(\mathrm{CH}_{3}\right)_{2}$

2, 8: $\mathrm{R}=p-\mathrm{Cl} ; \mathrm{R}^{1}=\mathrm{CH}\left(\mathrm{CH}_{3}\right)_{2}$

3, 9: $\mathrm{R}=p-\left(\mathrm{OCH}_{3}\right) ; \mathrm{R}^{1}=\mathrm{CH}\left(\mathrm{CH}_{3}\right)_{2}$

4, 10: $\mathrm{R}=\mathrm{H} ; \mathrm{R}^{1}=\mathrm{CH}_{2} \mathrm{CH}_{3}$

5, 11: $\mathrm{R}=p-\mathrm{Cl} ; \mathrm{R}^{1}=\mathrm{CH}_{2} \mathrm{CH}_{3}$

6, 12: $\mathrm{R}=p-\left(\mathrm{OCH}_{3}\right) ; \mathrm{R}^{1}=\mathrm{CH}_{2} \mathrm{CH}_{3}$

Scheme 1. Synthesis of 3,4-dialkyl-2,6-diaryl-piperidin-4-ol (7-12).

Among the two isomeric alcohols as shown in figure 1, the axial hydroxyl isomer was obtained as major isomer. Several attempts to obtain the equatorial hydroxyl isomer (minor product) with various eluting agents in various proportions were unsuccessful.
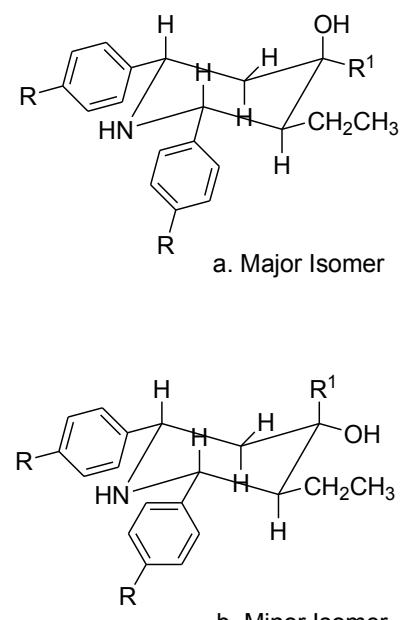

Figure 1. Isomeric forms of compounds 7-12. 
IR spectra reveal that the absence of absorption around $1700-1710 \mathrm{~cm}^{-1}$ corresponding to $\mathrm{C}=\mathrm{O}$ group and broad absorption around $1300-1400 \mathrm{~cm}^{-1}$ corresponding to $-\mathrm{NH}$ and $-\mathrm{OH}$ group clearly indicates the formation tertiary alcohol at $\mathrm{C}(4)$.

In ${ }^{1} \mathrm{H}-\mathrm{NMR}$, the aromatic protons are observed around $7 \mathrm{ppm}$. The $\mathrm{H}(2)$ and $\mathrm{H}(6)$ protons are resonated at 3.6-3.9 ppm (d) and 4.1-4.2 ppm (dd) respectively. As shown in table 1 , the observation of large coupling constant $(\sim 10 \mathrm{~Hz})$ and small coupling constant $\sim 2$ $3 \mathrm{~Hz}$ about $\mathrm{C}(5)-\mathrm{C}(6)$ bond reveals equatorial orientations of aryl rings at $\mathrm{C}(2)$ and $\mathrm{C}(6)$. Similarly, the observation of large coupling constant $(\sim 10 \mathrm{~Hz})$ about $\mathrm{C}(2)-\mathrm{C}(3)$ bond indicates the equatorial orientations of alkyl group at $\mathrm{C}(3)$. Hence, all the synthesized compounds exist in normal chair conformation with equatorial orientation of all the substituents.

Table 1. Coupling constant of Compounds $7 \mathbf{- 1 2}$.

\begin{tabular}{lllll}
\hline \multicolumn{5}{c}{ Coupling constant (In $H z)$} \\
\cline { 2 - 5 } Compound & ${ }^{\mathbf{3}} \mathbf{J}_{\mathbf{6 a}, \mathbf{5}}$ & ${ }^{\mathbf{3}} \mathbf{J}_{\mathbf{6} \mathbf{a}, \mathbf{e}}$ & ${ }^{2} \mathbf{J}_{\mathbf{5}, \mathbf{5 e}}$ & ${ }^{\mathbf{3}} \mathbf{J}_{\mathbf{2} \mathbf{a}, \mathbf{3}}$ \\
\hline 7 & 11.6 & 2.8 & 13.2 & 10.6 \\
9 & 12.8 & 3.2 & 12.8 & 10.4 \\
10 & 12.8 & 3.2 & 12.8 & 10.4 \\
11 & 10.25 & 2.25 & 12.75 & 10.0 \\
12 & 11.5 & 3.25 & 13.5 & 10.5 \\
\hline
\end{tabular}

The alkyl group at $\mathrm{C}(3)$ experiences severe gauche interaction with aryl group at $\mathrm{C}(2)$ and to avoid it, the ring flattened about $\mathrm{C}(2)-\mathrm{C}(3)$ bond. Hence, The coupling constant about $\mathrm{C}(2)-\mathrm{C}(3)$ bond $\left({ }^{3} \mathrm{~J}_{2 \mathrm{a}, 3 \mathrm{a}}\right)$ are considerably lower than the trans coupling constant about $\mathrm{C}(5)$ $\mathrm{C}(6)$ bond $\left({ }^{3} \mathrm{~J}_{6 \mathrm{a}, 5 \mathrm{a}}\right)$. The introduction of isopropyl group at $\mathrm{C}(4)$ instead of ethyl substituents increases ${ }^{3} \mathrm{~J}_{6 \mathrm{a}, 5 \mathrm{a}}$ due to ring puckering about $\mathrm{C}(5)-\mathrm{C}(6)$ bond.<smiles>CC1C2CC3(C)C(C)C(O2)C(C)C3C1C</smiles>

a

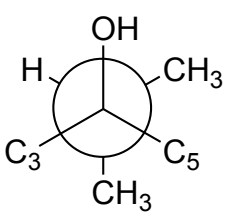

b<smiles>CC1C2CC3(C)C(C)C(C)C(C)(C2O)C13</smiles>

C

Figure 2. Conformation of C(4) substituent of 10-12. 
The compound 10-12 can exist in three possible conformation for the equatorial alkyl group and axial hydroxyl group at $\mathrm{C}(4)$ as shown in figure 2. The conformation $2 \mathrm{a}$ is destabilized due to severe gauche interaction. Hence it has been suggested that compound 10-12 exists as an equilibrium mixture of conformation 2a and 2c. However, syn-1,3-diaxial interaction among methyl group of isopropyl substituent at $\mathrm{C}(4)$ and adjacent benzylic proton, downfield signals were observed for $\mathrm{C}(3)$ and $\mathrm{C}(5)$ protons.

In ${ }^{13} \mathrm{C}-\mathrm{NMR}$, the signals at $62.9-66.0 \mathrm{ppm}$ and 56.2-62.0 were assigned to $\mathrm{C}(2)$ and $\mathrm{C}(6)$. The downfield absorption with low intensities at $68.1-74.4 \mathrm{ppm}$ were assigned to quaternary carbon, $\mathrm{C}(4)$. Also, introduction of isopropyl group shields $\mathrm{C}(4)$ carbon considerably due to electron donating nature. Also, the mass spectral data is consistent with the structure of compounds 7-12 as expected. It can be concluded that the substituents on $\mathrm{C}(4)$ position and substituted phenyl moiety were considerably influences the structural orientation.

\section{References}

1. Takahata H, Ouchi H, Ichinose M and Nemoto, H Org. Lett. 2002, 4 (20), 3459.

2. Honda T and Kimura M Org. Lett. 2000, 2 (24), 3925.

3. Aridoss G, Amirthaganesan S and Jeong Y T Bioorg. Med. Chem. Lett. 2010, 20, 2242.

4. Suleyman H, Inci Gul H and Alkan M Biol. Pharm. Bull. 2007, 30 (1), 63.

5. Balasubramanian S, Aridoss G, Parthiban P, Ramalingan $\mathrm{C}$ and Kabilan S Biol. Pharm. Bull. 2006, 29 (1), 125.

6. Jayabharathi J, Thangamani A, Padmavathy M and Krishnakumar B Med. Chem. Res. 2007, 15, 431.

7. Noller C R and Balaiah V J. Am. Chem. Soc. 1948, 70, 3853.

8. Manimekalai A, Maruthavanan T, Selvaraju K and Alkorta I J. Struct. Chem. 2007, 48 (6), 1036. 


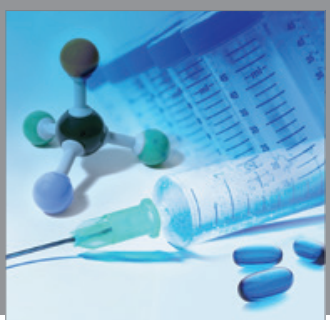

International Journal of

Medicinal Chemistry

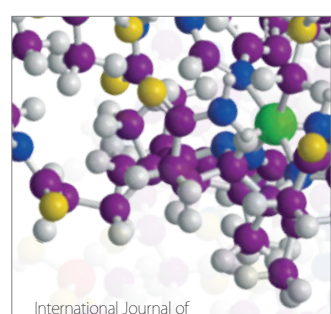

Carbohydrate Chemistry

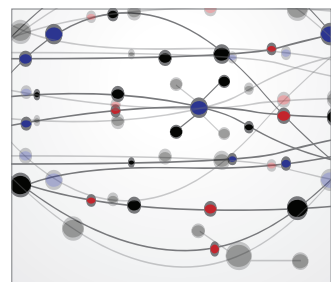

The Scientific World Journal
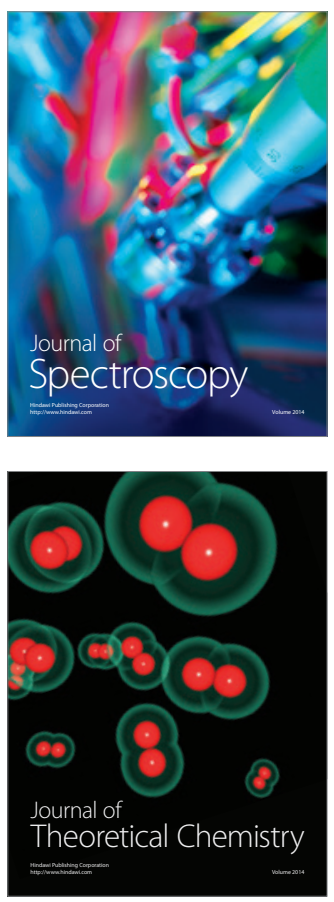
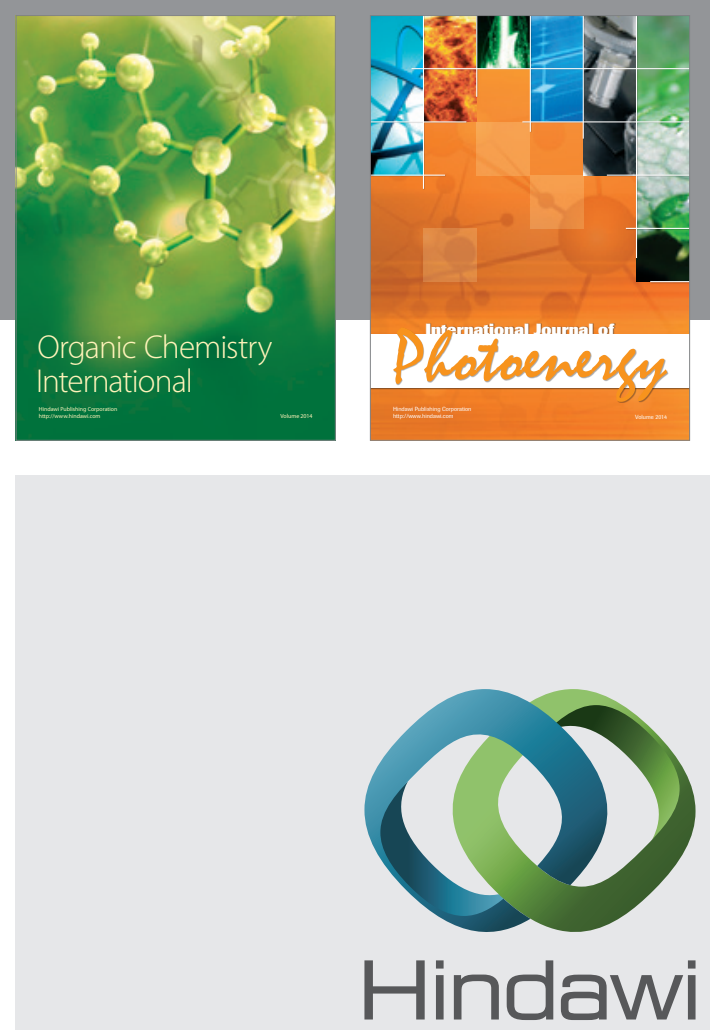

Submit your manuscripts at

http://www.hindawi.com
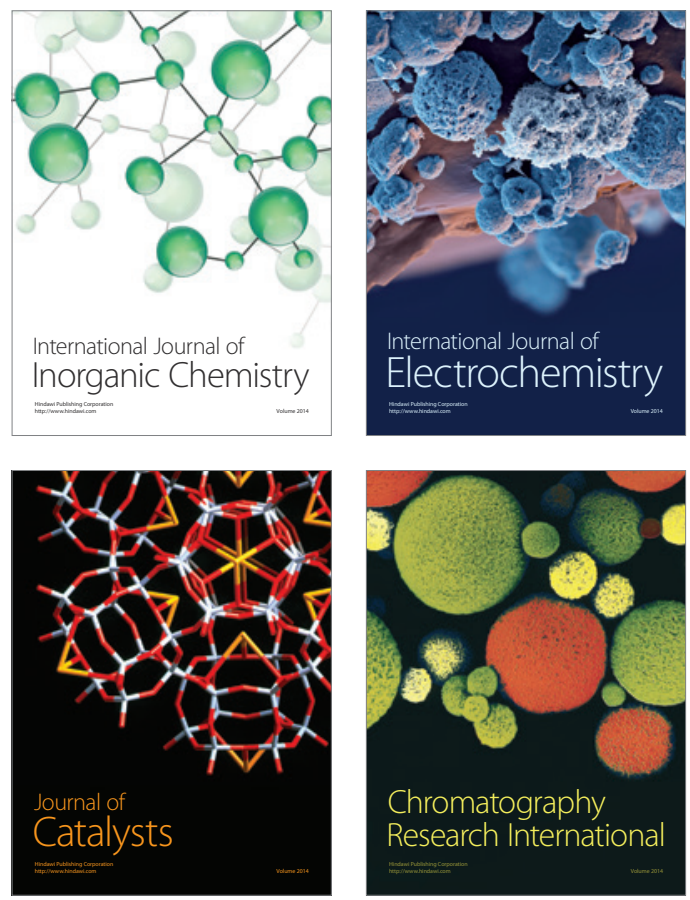
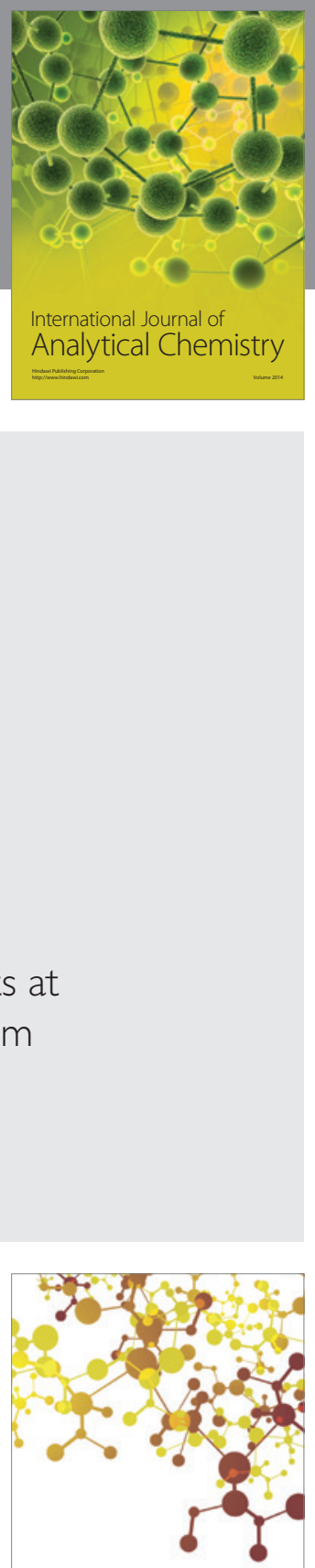

Journal of

Applied Chemistry
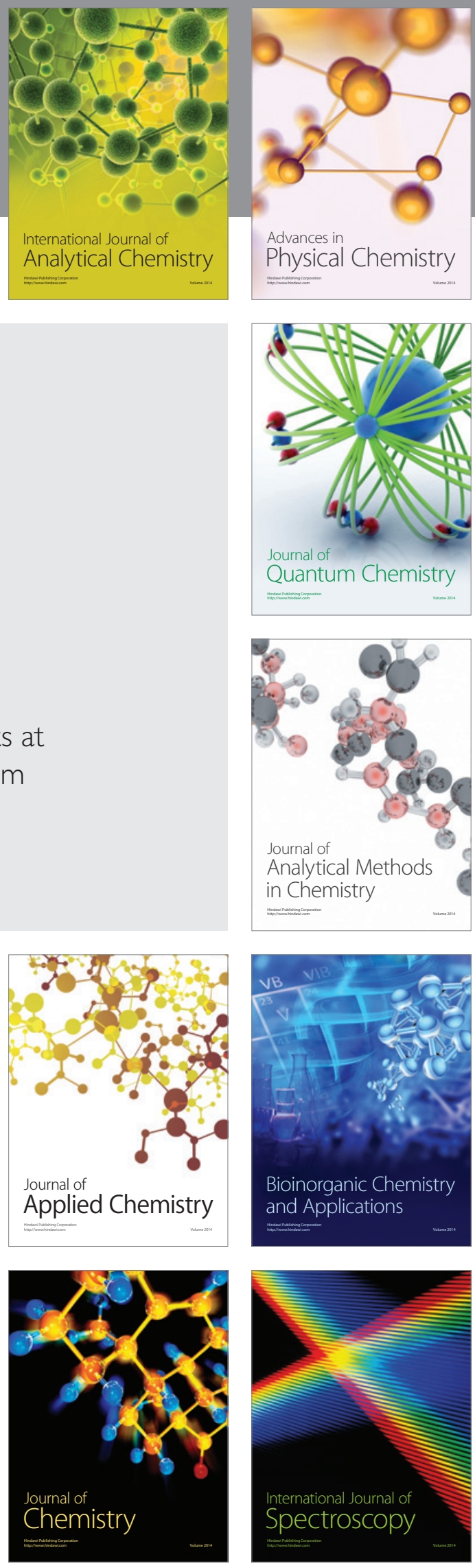